\title{
DISSOLVING A PARTNERSHIP EFFICIENTLY
}

\author{
By Peter Cramton, Robert Gibbons, and Paul Klemperer ${ }^{1}$
}

\begin{abstract}
Several partners jointly own an asset that may be traded among them. Each partner has a valuation for the asset; the valuations are known privately and drawn independently from a common probability distribution. We characterize the set of all incentive-compatible and interim-individually-rational trading mechanisms, and give a simple necessary and sufficient condition for such mechanisms to dissolve the partnership ex post efficiently. A bidding game is constructed that achieves such dissolution whenever it is possible. Despite incomplete information about the valuation of the asset, a partnership can be dissolved ex post efficiently provided no single partner owns too large a share; this contrasts with Myerson and Satterthwaite's result that ex post efficiency cannot be achieved when the asset is owned by a single party.
\end{abstract}

KEYWORDS: Mechanism design, efficient trading, fair division, auctions, public goods.

\section{INTRODUCTION}

WHEN A PARTNERSHIP IS TO BE DISSOLVED, who should buy out his associates and at what price? When municipalities jointly need a hazardous-waste dump, which town should provide the site and how much should it be compensated by the others? When husband and wife divorce, or children divide an estate, who should keep the family house or farm, and how much should the others be paid?

We consider partnerships in which each player $i$ is endowed with a share $r_{i}$ of a good to be traded, and specific capital or other transaction costs make it inefficient to sell the good on the market and split the proceeds. ${ }^{2}$ We look for procedures that allocate the good ex post efficiently while satisfying interim individual rationality. Unlike Myerson and Satterthwaite (1983) - who show that no procedure can yield both properties in two-player bargaining games with uncertainty $\left(r_{1}=1\right.$ and $\left.r_{2}=0\right)$-we show that the distributed ownership found in a partnership often makes the two compatible. For the case of $n$ players whose valuations are independently drawn from an arbitrary distribution, we derive a simple condition that is necessary and sufficient for efficient, individually-rational dissolution, and we introduce a simple bidding game that will accomplish such dissolution whenever it can be achieved as a Bayesian Nash equilibrium in some extensive-form game.

The application that inspired our analysis was the Federal Communication Commission's allocation of licenses for cellular-telephone franchises. After closing the list of applicants, the FCC proposed to make the final allocation using

\footnotetext{
${ }^{1}$ This project was begun at Stanford University and financially supported by the Sloan Foundation, the National Science Foundation, and the Center for Economic Policy Research. We are grateful to David Gold for posing the problem and encouraging its analysis, and to Roger Guesnerie, Christopher Harris, David Kreps, Robert Wilson, and two referees for helpful suggestions.

${ }^{2}$ See van Damme (1985) for a related study of fair division when each player has an equal ownership share.
} 
a simple lottery. Prior to the lottery each applicant has an equal chance of winning, and so can be thought of as owning a $1 / n$ share in the license. Our analysis suggests that the applicants would do better to form a cartel (that would win the lottery with certainty) and then allocate the franchise to one of their number via our bidding game; this is more efficient than the lottery, even if the winner is permitted to resell. A similar example is the Federal Aviation Administration's proposal to allocate landing slots at busy airports by lottery. Again, a more efficient approach is to assign the airlines shares in the slot equal to their weights in the proposed lottery, and let them play the bidding game we propose.

As another application of the theory, consider the following buy-out provision of many two-member partnerships: one side submits a "buy-out" offer, and the other side then has the choice of either buying or selling at these terms. Since this scheme does not guarantee ex-post efficiency (the first player will not, in general, submit his valuation, so the object will be inefficiently allocated if the other's valuation is between the first player's bid and valuation), it too can be improved upon by our bidding game.

The bidding game that achieves efficient, individually-rational dissolution differs from a typical auction in that every player pays or receives a sum of money that is a function of all the players' bids. Moreover, the set of bidders who pay a positive amount typically is not limited to the winning bidder, but includes the second and other high bidders as well. Since such bidding arrangements are not frequently observed, we determine the circumstances in which more common auctions-such as first- and second-price-can achieve efficient, individuallyrational dissolution. This part of our analysis was inspired by Samuelson (1985), who describes a similar problem and solves a two-player example in which types are drawn from a uniform distribution on $[0,1]$. He finds that split-the-difference bidding (the average of first- and second-price) yields an efficient allocation, but he ignores the individual rationality constraint that players should prefer participation in the game to retaining their current share. (He imposes instead the weaker constraint that players prefer participation to being dispossessed of their current share.) We consider $n$ players and arbitrary distributions and show that this and other similar auctions may accomplish efficient, individually-rational dissolution, but only when partners' shares are very close to equal.

Samuelson also provides an interesting interpretation of his work as an exploration of the Coase Theorem under incomplete information: instead of the complete-information conclusion that efficiency is always achieved and that property rights are immaterial, he shows that efficiency may be lost and property rights may matter. In these terms, our analysis shows exactly when efficiency can be achieved and how property rights matter.

The rest of the paper is organized as follows. In Sections 2 and 3, we analyze a revelation game to determine the set of partnerships that can be dissolved efficiently. Section 4 introduces a bidding game, that accomplishes efficient dissolution whenever it is possible, and Section 5 characterizes the set of partnerships for which efficient dissolution is possible. Section 6 shows that commonly observed auctions are efficient in some circumstances. 


\section{THE REVELATION GAME}

Our model has $n$ players indexed by $i \in N=\{1, \ldots, n\}$. Player $i$ owns a share $r_{i}$ of the good to be traded $\left(r_{i} \in[0,1]\right.$ and $\left.\sum_{i=1}^{n} r_{i}=1\right)$ and has a valuation for the entire good of $v_{i}$. Each player's valuation is known privately, but it is common knowledge that the valuations are drawn independently from a distribution $F$ with support $[\underline{v}, \bar{v}]$ and positive continuous density $f$.

We consider the direct revelation game in which players simultaneously report their valuations $v=\left\{v_{1}, \ldots, v_{n}\right\}$ and then receive an allocation $s(v)=$ $\left\{s_{1}(v), \ldots, s_{n}(v)\right\}$ and $t(v)=\left\{t_{1}(v), \ldots, t_{n}(v)\right\}$, where $s_{i}$ is the ownership share and $t_{i}$ is the net money transfer to player $i$. (Since this allocation is trader-specific, it may depend on the vector of initial ownership rights, $r=\left\{r_{1}, \ldots, r_{n}\right\}$.) We require that these allocations balance: $\sum s_{i}(v)=1$ and $\sum t_{i}(v)=0$ for all $v \in[\underline{v}, \bar{v}]^{n}$. The pair of outcome functions $\langle s, t\rangle$ is referred to as a trading mechanism.

A player with valuation $v_{i}$, share $r_{i}$, and money $m_{i}$ has utility $v_{i} r_{i}+m_{i}$, which is linear in money and the asset. Also, we assume that each player is endowed with enough money, say $\bar{v}$, that any required transfer is feasible. Because of the linear utility, only net transfers matter, so player $i$ 's utility before participating in the trading mechanism $\langle s, t\rangle$ can be taken to be $v_{i} r_{i}$, while afterwards it is $v_{i} s_{i}+t_{i}$. Let $-i=N \backslash i$ and let $\mathscr{E}_{-i}\{\cdot\}$ be the expectation operator with respect to $v_{-i}$. Then we can define the expected share and money transfer for player $i$ when he announces $v_{i}$ by

$$
S_{i}\left(v_{i}\right)=\mathscr{E}_{-i}\left\{s_{i}(v)\right\} \quad \text { and } \quad T_{i}\left(v_{i}\right)=\mathscr{E}_{-i}\left\{t_{i}(v)\right\},
$$

so the player's expected payoff is

$$
U_{i}\left(v_{i}\right)=v_{i} S_{i}\left(v_{i}\right)+T_{i}\left(v_{i}\right) .
$$

The mechanism $\langle s, t\rangle$ is incentive compatible if all types of all players want to report their private information truthfully:

$$
U_{i}\left(v_{i}\right) \geqslant v_{i} S_{i}(u)+T_{i}(u) \quad \forall i \in N, v_{i}, u \in[\underline{v}, \bar{v}] .
$$

By the Revelation Principle (Myerson (1979), among others), we lose no generality by restricting attention to incentive-compatible mechanisms. The mechanism $\langle s, t\rangle$ is interim individually rational if all types of all players are better off participating in the mechanism (in terms of their expected payoff) than holding their initial endowments:

$$
U_{i}\left(v_{i}\right) \geqslant r_{i} v_{i} \quad \forall i \in N \quad \text { and } \quad v_{i} \in[\underline{v}, \bar{v}] .
$$

The following lemmas develop a necessary and sufficient condition for a mechanism to be incentive compatible and individually rational. Since the proofs are either simple or standard, they are relegated to the Appendix.

LEMMA 1: The trading mechanism $\langle s, t\rangle$ is incentive compatible if and only if for every $i \in N, S_{i}$ is increasing and

$$
T_{i}\left(v_{i}^{*}\right)-T_{i}\left(v_{i}\right)=\int_{v_{i}^{*}}^{v_{i}} u d S_{i}(u)
$$

for all $v_{i}, v_{i}^{*} \in[\underline{v}, \bar{v}]$. 
Lemma 1 follows from the fact that utility is linear in money and the asset. Linearity implies that $U_{i}$ is convex and increasing in $v_{i}$, with derivative $S_{i}$ almost everywhere. The continuity of $U_{i}$ implies that the net utility $U_{i}\left(v_{i}\right)-r_{i} v_{i}$ has a minimum over $v_{i} \in[\underline{v}, \bar{v}]$. Lemma 2 identifies this worst-off type, and this allows us to restate individual rationality as a single condition in Lemma 3.

LEMMA 2: Given an incentive-compatible mechanism $\langle s, t\rangle$ trader $i$ 's net utility is minimized at $v_{i}^{*}=\frac{1}{2}\left[\inf \left(V_{i}^{*}\right)+\sup \left(V_{i}^{*}\right)\right] \in[\underline{v}, \bar{v}]$, where

$$
V_{i}^{*}=\left\{v_{i} \mid S_{i}(u)<r_{i} \forall u<v_{i} ; S_{i}(w)>r_{i} \forall w>v_{i}\right\} .
$$

In the simplest case, $S_{i}$ is continuous and has $r_{i}$ in its range, so the valuation of the worst-off type satisfies $S_{i}\left(v_{i}^{*}\right)=r_{i}$; that is, the worst-off type expects to receive a share equal to his initial ownership right $r_{i}$. Intuitively, the worst-off type expects on average to be neither a buyer nor a seller of the asset, and therefore he has no incentive to overstate or understate his valuation. Hence, he does not need to be compensated in order to induce him to report his valuation truthfully, which is why he is the worst-off type of trader.

This in an interesting generalization of a similar result in Myerson and Satterthwaite (1983) for bilateral exchange $(r=\{0,1\})$. In their paper, the lowest-type buyer $(\underline{v})$ and the highest-type seller $(\bar{v})$ are worst off; here the worst-off type typically is between $\underline{v}$ and $\bar{v}$, since it is no longer clear who is selling and who is buying.

LeMma 3: An incentive-compatible mechanism $\langle s, t\rangle$ is individually rational if and only if for all $i \in N$

$$
T_{i}\left(v_{i}^{*}\right) \geqslant 0 \text {, }
$$

where $v_{i}^{*}$ is defined in Lemma 2.

Lemmas 1-3 lead to a necessary and sufficient condition for a trading mechanism to be incentive compatible and individually rational, stated in Lemma 4 below.

LEMMA 4: For any share function $s$ such that $S_{i}$ is increasing for all $i \in N$, there exists a transfer function $t$ such that $\langle s, t\rangle$ is incentive compatible and individually rational if and only if

$$
\sum_{i=1}^{n}\left[\int_{v_{i}^{*}}^{\bar{v}}[1-F(u)] u d S_{i}(u)-\int_{\underline{v}}^{v_{t}^{*}} F(u) u d S_{i}(u)\right] \geqslant 0,
$$

where $v_{i}^{*}$ is defined in Lemma 2.

The "only if" part of the lemma follows directly from the previous lemmas and the budget balance conditions $\sum s_{i}(v)=1$ and $\sum t_{i}(v)=0$, which every feasible mechanism must satisfy. The "if" part of the lemma is proven by constructing a transfer function $t$ that is incentive compatible and individually rational provided 
the inequality (I) holds. The proof makes the following intuition precise: there exists a transfer rule $t$ that entices the worst-off type of each trader to participate in the mechanism, because (I) guarantees that the expected gains from trade are sufficient to bribe every trader to tell the truth.

\section{EX POST EFFICIENCY}

The trading mechanism $\langle s, t\rangle$ is ex post efficient if for each vector of valuations $v$ the outcome of the mechanism $\{s(v), t(v)\}$ is Pareto-undominated by any alternative allocation, ignoring incentive constraints. ${ }^{3}$ Thus, ex post efficiency requires that the asset go to the trader with the highest valuation. A partnership $\langle r, F\rangle$ can be dissolved efficiently if there exists an ex post efficient trading mechanism $\langle s, t\rangle$ that is incentive compatible and individually rational. Such a mechanism will be said to dissolve the partnership. For economy of expression, we will henceforth refer to ex post-efficient mechanisms that are incentive compatible and individually rational as efficient trading mechanisms. A partnership that can be dissolved efficiently will be referred to as a dissolvable partnership.

We are now prepared to answer the central question of this paper: What partnerships can be dissolved efficiently? At first glance, one might think that the set of dissolvable partnerships is empty; that is, the incomplete information about valuations necessarily leads to some inefficiency in trade. This is not the case. The following theorem gives a necessary and sufficient condition for the existence of an efficient trading mechanism.

THEOREM 1: A partnership with ownership rights $r$ and valuations independently drawn from $F$ can be dissolved efficiently if and only if

$$
\sum_{i=1}^{n}\left[\int_{v_{i}^{*}}^{\bar{v}}[1-F(u)] u d G(u)-\int_{\underline{v}}^{v_{i}^{*}} F(u) u d G(u)\right] \geqslant 0,
$$

where $v_{i}^{*}=F^{-1}\left(r_{i}^{1 / n-1}\right)$ and $G\left(v_{i}\right)=F\left(v_{i}\right)^{n-1}$.

Proof: Ex post efficiency requires that the good go to the trader who values it the most:

$$
s_{i}(v)=\left\{\begin{array}{lll}
0 & \text { if } & v_{i}<\max v_{j} \\
1 & \text { if } & v_{i}=\max v_{j}
\end{array}\right.
$$

(In the event that two or more traders have the highest valuation, then the shares can be split arbitrarily among them. Since ties occur with zero probability, they will be ignored in what follows.) By independence, the expected share function $S_{i}$ is given by

$$
S_{i}\left(v_{i}\right)=\operatorname{Pr}\left\{v_{i}>\max _{j \neq i} v_{j}\right\}=F\left(v_{i}\right)^{n-1}=G\left(v_{i}\right) .
$$

Thus, $v_{i}^{*}$ satisfies $F\left(v_{i}^{*}\right)^{n-1}=r_{i}$, so $v_{i}^{*}=F^{-1}\left(r_{i}^{1 / n-1}\right)$. Substituting into (I) of Lemma 4 yields (D).

\footnotetext{
${ }^{3}$ Our definition of ex post efficiency corresponds to classical ex post efficiency as defined in Holmstrom and Myerson (1983), since incentive constraints are ignored.
} 


\section{AN EFFICIENT BIDDING GAME}

In this section, we introduce a bidding game that serves the same purpose as an efficient trading mechanism. Using terminology analogous to that introduced in Section 3, given a dissolvable partnership, one could use an efficient trading mechanism or an efficient bidding game to dissolve it. This is a useful complement to the revelation-game analysis of Section 2 , because it uses strategy spaces familiar in practice, namely bids rather than valuations. In a general bidding game, the $n$ players submit sealed bids, the good is transferred to the highest bidder, and each bidder $i$ pays a total price $P_{i}\left(b_{1}, \ldots, b_{n}\right)$. In the efficient bidding game analyzed below, the total price $P_{i}$ is the sum of a price

$$
p_{i}\left(b_{1}, \ldots, b_{n}\right)=b_{i}-\frac{1}{n-1} \sum_{j \neq i} b_{j}
$$

and a side-payment $c_{i}$ that can precede the bidding. Note that in the efficient bidding game the winning bidder pays a positive price $p_{i}$, as usual, but so may the second and other high bidders. As in a standard auction, a higher bid buys the player a larger probability of winning. Here, however, making a higher bid is like buying more lottery tickets in that the purchase price of losing tickets is not refunded.

THEOREM 2: A bidding game with prices

$$
p_{i}\left(b_{1}, \ldots, b_{n}\right)=b_{i}-\frac{1}{n-1} \sum_{j \neq i} b_{j}
$$

preceded by side-payments

$$
c_{i}\left(r_{1}, \ldots, r_{n}\right)=\int_{\underline{v}}^{v_{1}^{*}} u d G(u)-\frac{1}{n} \sum_{j=1}^{n} \int_{\underline{v}}^{v_{j}^{*}} u d G(u),
$$

is an efficient bidding game: it dissolves any dissolvable partnership.

Proof: We solve for a strictly increasing symmetric Bayesian equilibrium. If the $n-1$ others use the strategy $b(\cdot)$, then $i$ 's expected utility from bidding $b_{i}$ with valuation $v_{i}$ is

$$
\begin{aligned}
U_{i}\left(v_{i}, b_{i}\right)= & \int_{\underline{v}}^{b^{-1}\left(b_{i}\right)}\left[v_{i}-b_{i}+\frac{1}{n-1} b(u)+\frac{n-2}{n-1} \vec{b}(u)\right] d G(u) \\
& +\int_{b^{-1}\left(b_{i}\right)}^{\bar{v}}\left[-b_{i}+\frac{1}{n-1} b(u)+\frac{n-2}{n-1} \bar{b}(u)\right] d G(u),
\end{aligned}
$$

where $\bar{b}(u)=\int_{\underline{v}}^{u} b\left(v_{j}\right) d F\left(v_{j} \mid u\right)$ and $F\left(v_{j} \mid u\right)=F\left(v_{j}\right) / F(u)$. (Since types are independent, all but the highest of the $n-1$ other bids generate the same expected value, conditional on the value of the highest bid; this is $\bar{b}(u)$.) The best response for $i$ therefore solves

$$
\frac{\partial U_{i}}{\partial b_{i}}=-1+\frac{d b^{-1}}{d b_{i}} v_{i} g\left[b^{-1}\left(b_{i}\right)\right]=0 .
$$


Since $\partial U_{i} / \partial b_{i}$ is positive (negative) for $b_{i}$ less than (greater than) $b\left(v_{i}\right)$, the second-order condition is satisfied. We are interested in the symmetric solution, which satisfies

$$
b\left(v_{i}\right)=\int_{u=\underline{v}}^{v_{t}} u d G(u)+b(\underline{v}) .
$$

Since $b^{\prime}>0$, this equilibrium is ex post efficient: the trader with the highest valuation receives the good. The constant $b(\underline{v})$ is arbitrary (it equals the lowest amount, presumably zero, that the rules of the game allow a player with valuation $\underline{v}$ to bid), and disappears when $p_{i}$ and $b$ are composed:

$$
p_{i}\left[b\left(v_{1}\right), \ldots, b\left(v_{n}\right)\right]=-\int_{\underline{v}}^{v_{t}} u d G(u)+\frac{1}{n-1} \sum_{j \neq i} \int_{\underline{v}}^{v_{j}} u d G(u) .
$$

Some simple algebra verifies that $(\mathrm{T})$ and $(\mathrm{C})$ define the transfer rule used in the "if" part of the proof of Lemma 4 , so individual rationality is guaranteed.

Q.E.D.

Since the side-payments depend on $r=\left\{r_{1}, \ldots, r_{n}\right\}$ (through $v^{*}=\left\{v_{1}^{*}, \ldots, v_{n}^{*}\right\}$ ) and $F$, but not on $v=\left\{v_{1}, \ldots, v_{n}\right\}$, they can precede the bidding procedure. Their purpose is to compensate large shareholders, who are effectively dispossessed in the bidding game that follows, since the prices $p_{i}$ are independent of $r$ and so treat all shareholders alike. Accordingly, the side-payments are zero for the equal-shares partnership $(1 / n, \ldots, 1 / n)$.

\section{CHARACTERIZATION RESULTS}

We now offer four propositions that characterize the set of partnerships which can be dissolved efficiently. The proofs are not of interest in themselves, and so are given in the Appendix. First, we formalize the idea that it is large shareholders that make interim individual rationality difficult to achieve: for any distribution $F$, the equal-share partnership is dissolvable but the partnership in which one player owns the entire asset is not.

Proposition 1: The set of partnerships that can be dissolved efficiently is a nonempty, convex, symmetric subset of the $n-1$ dimensional simplex and is centered around the equal-shares partnership $(1 / n, \ldots, 1 / n)$.

Proposition 2: A one-owner partnership $\left\{r_{1}=1, r_{2}=0, \ldots, r_{n}=0\right\}$ cannot be dissolved efficiently.

Proposition 2 generalizes to many buyers Myerson and Satterthwaite's (1983) result that a buyer-seller relationship cannot simultaneously satisfy ex post efficiency and interim individual rationality. This speaks to the time-honored tradition of solving complex allocation problems by resorting to lotteries: even if the winner is allowed to resell the object, such a scheme is inefficient because 
the one-owner partnership that results from the lottery cannot be dissolved efficiently.

These propositions are derived by making the appropriate substitutions into (D) of Theorem 1 . Note that each partner's ownership share $r_{i}$ enters the inequality through $v_{i}^{*}$.

As an example, if the traders' valuations are drawn from a uniform distribution on $[0,1]$, then $(D)$ simplifies to

$$
\sum_{i=1}^{n} r_{i}^{n / n-1} \leqslant \frac{n}{n+1}
$$

Thus, for a uniform distribution, a partnership $r$ can be dissolved efficiently if and only if $\left(D^{\prime}\right)$ is satisfied. By Proposition $1,\left(D^{\prime}\right)$ determines a convex, symmetric subset of the simplex, shown as the unshaded region in Figure 1 for the case $n=3$. Only partnerships in the extremities of the simplex cannot be dissolved efficiently. For the uniform case, as the number of partners $(n)$ grows, the percentage of partnerships that are dissolvable increases from $58 \%$ to $93 \%$ to $99 \%$ as $n$ increases from 2 to 4 to 6 . Also, the percentage share of the largest possible owner in a dissolvable partnership increases from $79 \%$ to $82 \%$ to $88 \%$ as $n$ increases from 2 to 20 to 200 .

By contrast with Proposition 2, however, partnerships with an arbitrarily small amount of distributed ownership may be dissolvable. (Note that the proof employs a very special distribution.)

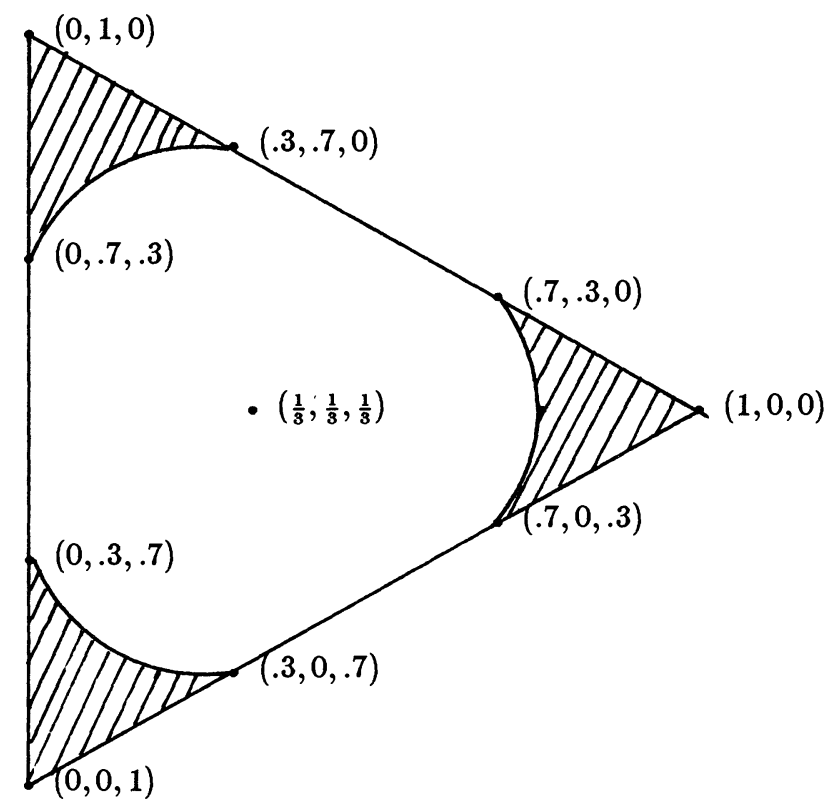

FIGURE 1-Dissolvable partnerships with $n=3$ and $F(u)=u$. 
Proposition 3: Any partnership not owned by a single player can be dissolved efficiently for some distributions $F$.

Finally, if any partnership is replicated a sufficient number of times then it can be dissolved efficiently. Consider the partnership

$$
R(n \mid m)=\left\{r_{i}=1 / m, i=1, \ldots, m ; r_{j}=0, j=m+1, \ldots, n m\right\} .
$$

This partnership results from replicating the $n$-player, one-owner partnership $m$ times-the $m$ partners who own positive shares each own $1 / m$ of the total endowment of $m$ goods, so partnerships continue to be represented by points in a simplex. The $m$-fold replication of any other $n$-player partnership can be represented in a similar way.

Proposition 4: Given $F$ and an n-player partnership $\left\{r_{1}, \ldots, r_{n}\right\}$, there exists a finite $M$ such that for all $m>M$ the $m$-fold replication of the n-player partnership can be dissolved efficiently.

We think of this result as complementing the core-convergence theorems for exchange economies: replicating the economy sufficiently often reduces the effect of the incomplete information to zero.

An interesting special case is $R(2 \mid m)$. For $m=1$, Myerson and Satterthwaite's result (and our Proposition 2 above) proves that the partnership cannot be dissolved efficiently. For larger values of $m, R$ is much like the double auction studied by Wilson (1985) and Gresik and Satterthwaite (1985) although there each bidder wants only one unit of the good, whereas here each bidder may demand up to $m$ units of the good. (Think of each $1 / \mathrm{m}$ share of the partnership as one unit of the good.) When each bidder wants one unit, Gresik and Satterthwaite show that ex post efficiency is approached in the limit; more specifically, for the uniform case, they find that 99.31 per cent of the gains from trade are realized if there are six traders on each side of the market. When each bidder wants $m$ units, on the other hand, ex post efficiency is achieved for the same example when there are as few as two traders on each side of the market. (To see this, check that $\left(\mathrm{D}^{\prime}\right)$ holds for $R(2 \mid 2)$ for this example.)

\section{SIMPLE TRADING RULES}

The efficient bidding game proposed in Theorem 2 dissolves any dissolvable partnership. Although the efficient trading mechanism implicit in Lemma 4 and Theorem 1 achieves the same effect, we prefer the bidding game for two (somewhat imprecise) reasons. First, as mentioned above, it uses strategy spaces familiar in practice. And second, and probably more important, in the bidding game a great deal of the computational burden has been shifted from the mechanism designer to the players: the designer makes a simple calculation of side-payments and prices, using $(\mathrm{C})$ and $(\mathrm{P})$, while the players do most of the work in their calculation of the optimal bidding strategy $b\left(v_{i}\right)$. In the trading mechanism, on the other hand, the players simply report their valuations while the designer shoulders all of the computational burden. 
In this spirit, we find it disappointing that the side-payments given by $(C)$ in Theorem 2 depend on the distribution $F$, for it seems plausible that the designer will know $F$ less well than do the players. Ideally we would like a bidding game that can be described independently of $F$, so that the designer could always recommend it without assessing the distribution of the partners' private valuations. Unfortunately, the Revelation Principle is no help here: it analyzes the composition of the players' strategies and the designer's game rules, but gives no guidance as to how to decompose this map from types to outcomes into strategies for the players that may depend on $F$ and game rules for the designer that are independent of $F$.

We have not addressed the issue of finding the optimal bidding game or trading mechanism that is independent of $F$, in part because of the difficulty in formalizing this notion. (Asking only for independence of $F$, for instance, is not enough, because the designer can ask the players to report $F$, and then implement the side-payments given by $(C)$ if the reports agree, and forbid trade if the reports do not agree.) Instead, we offer two speculative approaches to bidding games that are independent of $F$, in the hope that these ideas will be expanded upon.

First, it may be possible for the designer to use the players' bids to estimate $F$, and then to use this estimate to construct side-payments that relax the individual rationality constraints, like those in $(\mathrm{C})$. This process seems both complex and delicate. $^{4}$

Second, some bidding games that are independent of $F$ can dissolve a limited subset of the set of dissolvable partnerships. In particular, the game we discuss below dissolves the equal-shares partnership for any distribution $F$. In addition, this bidding game has three other virtues when compared to the efficient bidding game in Theorem 2. First, it is simple and familiar. Second, it is less vulnerable to collusion. (In the efficient bidding game, a cartel saves the cost of all losing bids by submitting only one nonzero bid.) And third, it relies less heavily on the risk neutrality of the bidders, since only the winner is required to pay.

Specifically, we consider a " $k+1$-price auction" in which the players submit sealed bids and the good is transferred to the highest bidder, who pays each of the others

$$
p\left(b_{1}, b_{2}, \ldots, b_{n}\right)=\frac{1}{n}\left[k b_{s}+(1-k) b_{f}\right]
$$

\footnotetext{
${ }^{4}$ More precisely, estimating $F$ seems complex and using the estimate seems delicate. As an example, suppose that $F$ is known to be approximately uniform on $[0, \bar{v}]$ with $\bar{v} \in[\underline{v}, \bar{V}]$. Consider playing the bidding game of Theorem 2 without the side-payments. Then for every pair of players $\{i, j\}$, use the remaining $n-2$ players' bids to estimate $F$, as follows. First, use the symmetric equilibrium bidding strategy identified in the text to map an arbitrary distribution of valuations $F$ into a distribution of bids. And second, vary $F$ over the set of distributions described above in order to maximize some goodness-of-fit criterion imposed on the two distributions of bids, one observed, and the other calculated. Now use this estimate to calculate the side-payment $c_{j}\left(r_{1}, \ldots, r_{n}\right)$ given in (C) and let $i$ pay $j$ the amount $c_{j} /(n-1)$. In this construction, the payments received by any one player depend only on the other players' bids, so the equilibrium strategies are unaffected. Therefore, the size of the subset of the set of dissolvable partnerships that can be dissolved in this way depends only on how well these elaborately constructed side-payments mimic those defined by $(C)$ when $F$ is known.
} 
where $b_{f}$ and $b_{s}$ are the first- and second-highest bids from $\left\{b_{1}, b_{2}, \ldots, b_{n}\right\}$, and $k \in[0,1]$. For $k=0$ this is like a first-price auction, for $k=1$ it is like a second-price auction, and for $k=\frac{1}{2}$ it is the split-the-difference scheme described by Samuelson (1985).

Note that the revenue from bidding (namely, the highest bidder's bid) is divided equally among all the bidders. This is important. When the original ownership shares are unequal, the individual rationality constraints could be more easily met by paying losing bidders in proportion to their shares, but then partners owning different shares would have different equilibrium bidding strategies so the partner with the highest valuation might not win, violating ex post efficiency. ${ }^{5}$

We begin by calculating an equilibrium bidding strategy for this auction. Calculating the interim expected utility associated with this equilibrium then determines the set of partnerships that can be dissolved efficiently using the $k+1$-price auction. (Again, the terminology is analogous to that introduced in Section 3.)

Proposition 5: $A k+1$-price auction has a symmetric equilibrium bidding strategy given by

$$
b\left(v_{i}\right)=v_{i}-\frac{\int_{z=F^{-1}(k)}^{v_{i}}[F(z)-k]^{n} d z}{\left[F\left(v_{i}\right)-k\right]^{n}} .
$$

Proposition 6: The set of partnerships that can be dissolved efficiently using a $k+1$ price auction is a nonempty, convex, symmetric subset of the simplex and is centered around the equal-shares partnership $(1 / n, \ldots, 1 / n)$.

Thus, an equal-shares partnership can always be dissolved efficiently by any $k+1$-price auction. Such a simple auction only works, however, when partners' shares are approximately equal, since the auction ignores the ownership rights $r$ and this makes large shareholders unwilling to participate. For the uniform case, the $k+1$-price auction dissolves a partnership if and only if $\left(\max r_{i}\right)^{n / n-1} \leqslant$ $1 /(n-1)$, which for $n=2, n=20$, and $n=200$ is satisfied if no partner's share exceeds $57.7 \%, 5.54 \%$ and $0.511 \%$, respectively. (A special property of the uniform distribution is that these results are independent of $k$. Contrast these results, however, with the corresponding results for the efficient bidding game, which are given after Proposition 2.) The intuition is that, because the auction treats all players as if they owned share $1 / n$, large shareholders will participate only if the expected gain from trade exceeds the cost of being, in effect, dispossessed. As $n$ increases, the expected gain from trade of the worst-off type decreases: a player with high share and high valuation becomes almost certain to be just outbid by players with slightly higher valuations. Thus given a share $\rho \in[0,1]$ there will exist some $N_{\rho}$ such that a partner with share $\rho$ will be willing to participate only if $n \leqslant N_{\rho}$, and in the limit, only shareholders with $\rho \leqslant 1 / n$ are willing to participate.

\footnotetext{
${ }^{5}$ We could for every partner $j$ divide $j$ 's bid among the other $(n-1)$ players in proportion to the other's relative shares, since then incentives for the bidders are unaffected by their relative shares. This is an example of the type of auction discussed in the previous footnote and would typically perform better than the auction considered here, at some cost in terms of greater complexity.
} 
Proposition 7: As $n \rightarrow \infty$, the only partnership that can be dissolved efficiently by a $k+1$-price auction is the equal-shares partnership $(1 / n, \ldots, 1 / n)$. That is, letting $\rho_{n}$ be the largest share in a n-player partnership that any partner can have such that the partnership can be dissolved efficiently, $\rho_{n} /(1 / n) \rightarrow 1$ as $n \rightarrow \infty$.

\section{CONCLUSION}

A simple extension of Myerson and Satterthwaite (1983) shows that with incomplete information no mechanism can guarantee that an object to be traded will be allocated to the person who values it most, if the object is initially owned by a single party. In contrast, we show that if the ownership is distributed among a partnership, ex-post efficient allocation is often possible. Further, when it is possible, it can be achieved by a simple bidding game. In a more general model of partnerships, our observation that the range of partnerships that can be dissolved efficiently is centered around equal shares suggests that this might be a factor influencing the way in which partnerships are formed.

Yale School of Management, Box 1A, Yale Station, New Haven, CT 06520, U.S.A.,

Dept. of Economics, Massachusetts Institute of Technology, Cambridge, MA 02139, U.S.A.,

St. Catherines College and The Institute of Economics and Statistics, Oxford University, U.K.

Manuscript received October, 1984; final revision received July, 1986.

\section{APPENDIX}

This Appendix supplies the proofs of Lemmas 1-4 and Propositions 1-7.

LEMMA 1: The trading mechanism $\langle s, t\rangle$ is incentive compatible if and only if for every $i \in N, S_{1}$ is increasing and

$$
T_{1}\left(v_{i}^{*}\right)-T_{1}\left(v_{1}\right)=\int_{v_{i}^{*}}^{v_{\imath}} u d S_{i}(u)
$$

for all $v_{i}, v_{1}^{*} \in[\underline{v}, \bar{v}]$.

Proof: Only If. If $\langle s, t\rangle$ is incentive compatible, then $U_{l}\left(v_{1}\right)=v_{t} S_{i}\left(v_{t}\right)+T_{i}\left(v_{t}\right) \geqslant v_{t} S_{l}(u)+T_{1}(u)$, or equivalently

$$
U_{l}\left(v_{\imath}\right) \geqslant U_{l}(u)+\left(v_{t}-u\right) S_{1}(u),
$$

implying that $U_{1}$ has a supporting hyperplane at $u$ with slope $S_{i}(u) \geqslant 0$. Thus, $U_{t}$ is convex and has derivative $d U_{i} / d v_{i}=S$ almost everywhere. Also, $S$, must be increasing, and

$$
U_{l}\left(v_{\imath}\right)-U_{\imath}\left(v_{\imath}^{*}\right)=\int_{v_{i}^{*}}^{v_{\imath}} S_{l}(u) d u
$$


(We use the Stieltjes integral throughout this paper, so that any discontinuities in the expected share function are accounted for in the integral.) By integration by parts,

$$
\int_{v_{i}^{*}}^{v_{1}} S_{i}(u) d u=v_{1} S_{i}\left(v_{1}\right)-v_{i}^{*} S_{i}\left(v_{i}^{*}\right)-\int_{v_{i}^{*}}^{v_{\imath}} u d S_{\imath}(u),
$$

which together with the definition of $U_{l}$ yields (IC).

If. Adding the identity

$$
v_{i}\left[S_{i}\left(v_{i}\right)-S_{i}\left(v_{i}^{*}\right)\right]=v_{i} \int_{v_{i}^{*}}^{v_{i}} d S_{i}(u)
$$

to (IC) results in

$$
v_{i}\left[S_{\imath}\left(v_{\imath}\right)-S_{t}\left(v_{\imath}^{*}\right)\right]+T_{i}\left(v_{\imath}\right)-T_{\imath}\left(v_{i}^{*}\right)=\int_{v_{i}^{*}}^{v_{\imath}}\left(v_{i}-u\right) d S_{i}(u) \geqslant 0,
$$

where the inequality follows because the integrand is nonnegative for all $v_{\imath}, u \in[\underline{v}, \bar{v}]$, since $S_{t}$ is increasing. Rearranging the terms on the left-hand side yields

$$
v_{t} S_{i}\left(v_{\imath}\right)+T_{i}\left(v_{i}\right) \geqslant v_{i} S_{i}\left(v_{i}^{*}\right)+T_{t}\left(v_{t}^{*}\right),
$$

which is incentive compatibility.

LEMMA 2: Given an incentive-compatible mechanism $\langle s, t\rangle$, trader i's net utility is minimized at $v_{i}^{*}=\frac{1}{2}\left[\inf \left(V_{i}^{*}\right)+\sup \left(V_{i}^{*}\right)\right] \in[\underline{v}, \bar{v}]$, where

$$
V_{\imath}^{*}=\left\{v_{\imath} \mid S_{\imath}(u)<r_{i} \forall u<v_{i} ; S_{i}(w)>r_{i} \forall w>v_{\imath}\right\} .
$$

PROof: The net utility to trader $i$ with valuation $v_{i}$ is $U_{i}\left(v_{i}\right)-r_{i} v_{i}$, which is convex in $v_{i}$ by Lemma 1. Therefore, trader $i$ 's net utility is minimized at the point where the left and right derivatives of $U_{t}$ with respect to $v_{l}$ bound $r_{i}$. But $d U_{i} / d v_{l}=S_{1}$ almost everywhere, $S_{1}$ is increasing, and $T_{i}$ is decreasing in $v_{i}$. Four cases need to be considered. First, suppose that $S_{t}(u)>r_{i}$ or $S_{i}(u)<r_{t}$ for all $u \in[\underline{v}, \bar{v}]$; then the minimum occurs at the boundaries $v_{i}^{*}=\underline{v}$ or $v_{i}^{*}=\bar{v}$, respectively. The next three cases deal with the case where there exists $u$ and $w$ such that $S_{t}(u)>r_{t}$ and $S_{i}(w)<r_{r}$. (1) Suppose $S_{i}$ is continuous and strictly increasing; then there exists a unique $v_{i}^{*}$ such that $S_{i}\left(v_{i}^{*}\right)=r_{i}$, which minimizes trader $i$ 's net utility. (2) If $S_{i}$ is not continuous and $S_{1}$ jumps past $r_{i}$, then the $v_{i}$ at which $S_{1}$ jumps minimizes net utility. (3) Finally, if $S_{i}(u)=r_{i}$ over an interval, then each type in the interval is equally worse off and we can arbitrarily select any valuation in the interval to be the worst-off type.

Q.E.D.

LEMMA 3: An incentive-compatible mechanism $\langle s, t\rangle$ is individually rational if and only if for all $i \in N$

$$
T_{i}\left(v_{i}^{*}\right) \geqslant 0,
$$

where $v_{i}^{*}$ is defined in Lemma 2.

Proof: We need only check individual rationality at the valuation $v_{l}^{*}$ defined in Lemma 2 . Thus, the individual-rationality constraint becomes $v_{i}^{*} S_{i}\left(v_{i}^{*}\right)+T_{i}\left(v_{i}^{*}\right) \geqslant r_{i} v_{i}^{*}$, or $r_{i} v_{i}^{*}+T_{i}\left(v_{i}^{*}\right) \geqslant r_{i} v_{i}^{*}$.

Q.E.D.

LEMMA 4: For any share function $s$ such that $S_{1}$ is increasing for all $i \in N$, there exists a transfer function $t$ such that $\langle s, t\rangle$ is incentive compatible and individually rational if and only if

$$
\sum_{i=1}^{n}\left[\int_{v_{i}^{*}}^{\bar{v}}[1-F(u)] u d S_{\imath}(u)-\int_{\underline{v}}^{v_{i}^{*}} F(u) u d S_{i}(u)\right] \geqslant 0,
$$

where $v_{\imath}^{*}$ is defined in Lemma 2.

Proof: Only if. Suppose $\langle s, t\rangle$ is incentive compatible and individually rational. Then from Lemma 1,

$$
T_{1}\left(v_{\imath}\right)=T_{\imath}\left(v_{i}^{*}\right)-\int_{v_{i}^{*}}^{v_{1}} u d S_{\imath}(u)
$$


Integrating over all types in $[\underline{v}, \bar{v}]$ yields

$$
\begin{aligned}
\mathscr{E}_{i}\left\{T_{\imath}\left(v_{i}\right)\right\} & =T_{i}\left(v_{i}^{*}\right)-\int_{v_{t}=y}^{\bar{u}} \int_{u=v_{t}^{*}}^{v_{\imath}} u d S_{\imath}(u) d F\left(v_{\imath}\right) \\
& =T_{i}\left(v_{i}^{*}\right)-\int_{u=v_{t}^{*}}^{\bar{v}} \int_{v_{\imath}=u}^{\bar{u}} d F\left(v_{i}\right) u d S_{\imath}(u)+\int_{u=v}^{v_{t}^{*}} \int_{v_{t}=v}^{u} d F\left(v_{i}\right) u d S_{\imath}(u) \\
& =T_{\imath}\left(v_{\imath}^{*}\right)-\int_{v_{t}^{*}}^{\bar{v}}[1-F(u)] u d S_{\imath}(u)+\int_{v}^{v_{t}^{*}} F(u) u d S_{i}(u),
\end{aligned}
$$

where the second line follows from changing the order of integration. Budget balance requires $\sum_{i=1}^{n} t_{i}(v)=0$ for all $v$, so we have

$$
\sum_{i=1}^{n} \mathscr{C}_{i}\left\{T_{i}\left(v_{1}\right)\right\}=\mathscr{E}_{N}\left\{\sum_{i=1}^{n} t_{i}(v)\right\}=0 .
$$

Therefore, summing over all traders yields

$$
\sum_{i=1}^{n} T_{i}\left(v_{i}^{*}\right)=\sum_{i=1}^{n}\left[\int_{v_{i}^{*}}^{\bar{v}}[1-F(u)] u d S_{i}(u)-\int_{v}^{v_{i}^{*}} F(u) u d S_{i}(u)\right] .
$$

From Lemma 3, $T_{i}\left(v_{i}^{*}\right)$ must be nonnegative for all $i$, which implies $\sum_{i=1}^{n} T_{i}\left(v_{i}^{*}\right) \geqslant 0$.

If. The proof is by construction. Let

$$
t_{i}(v)=c_{i}-\int_{\underline{v}}^{v_{1}} u d S_{\imath}(u)+\frac{1}{n-1} \sum_{j \neq i} \int_{v}^{v_{j}} u d S_{j}(u),
$$

where $\sum_{t=1}^{n} t_{i}(v)=0$ implies $\sum_{t=1}^{n} c_{t}=0$. Then, after changing the order of integration,

$$
T_{\imath}\left(v_{i}\right)=c_{i}-\int_{\underline{v}}^{v_{1}} u d S_{\imath}(u)+\frac{1}{n-1} \sum_{j \neq i} \int_{\underline{v}}^{\bar{v}}[1-F(u)] u d S_{j}(u),
$$

so Lemma 1 guarantees that $\langle s, t\rangle$ is incentive compatible. Finally, by Lemma 3 , we have individual rationality if and only if $T_{i}\left(v_{i}^{*}\right) \geqslant 0$. A little algebra shows the hypothesis of Lemma 4 to be equivalent to the condition $\sum_{i=1}^{n} T_{i}\left(v_{l}^{*}\right) \geqslant 0$, so we can choose

$$
c_{i}=\frac{1}{n} \sum_{i=1}^{n} T_{i}\left(v_{i}^{*}\right)+\int_{\underline{v}}^{v_{i}^{*}} u d S_{i}(u)-\frac{1}{n-1} \sum_{j \neq i} \int_{\underline{v}}^{\bar{v}}[1-F(u)] u d S_{j}(u),
$$

which results in $T_{i}\left(v_{i}^{*}\right)=(1 / n) \sum_{i=1}^{n} T_{i}\left(v_{i}^{*}\right) \geqslant 0$.

Q.E.D.

Proposition 1: The set of partnerships that can be dissolved efficiently is a nonempty, convex, symmetric subset of the n-1-dimensional simplex and is centered around the equal shares partnership $(1 / n, \ldots, 1 / n)$.

Proof: Use (D) to define $\phi: \mathscr{R}^{n} \rightarrow \mathscr{R}$ by

$$
\phi(r)=\sum_{i=1}^{n}\left[\int_{v_{i}^{*}}^{\bar{v}} v_{l} f\left(v_{i}\right) F\left(v_{l}\right)^{n-2} d v_{l}-\int_{\underline{v}}^{\bar{v}} v_{i} f\left(v_{l}\right) F\left(v_{i}\right)^{n-1} d v_{l}\right] .
$$

Convexity follows from concavity of $\phi$, which we have because

$$
\begin{aligned}
& \frac{\partial \phi}{\partial r_{t}}=\frac{-v_{t}^{*} d F\left(v_{t}^{*}\right)^{n-1}}{n-1} \cdot \frac{d v_{i}^{*}}{d r_{t}}=\frac{-v_{i}^{*}}{n-1}, \\
& \frac{\partial^{2} \phi}{\partial r_{t} \partial r_{l}}=0, \quad \text { and } \quad \frac{\partial^{2} \phi}{\partial r_{t}^{2}}=-\frac{1}{n-1} \cdot \frac{d v_{i}^{*}}{d r_{t}}<0 .
\end{aligned}
$$

Symmetry follows from relabeling the partners. Finally, $\phi(1 / n, \ldots, 1 / n)>0$ because at $v_{l}^{*}(1 / n)=$ $F^{-1}\left(1 / n^{1 / n-1}\right)$ we have

$$
\begin{aligned}
\int_{v_{i}^{*}}^{\bar{v}} v_{l} f\left(v_{\imath}\right) F\left(v_{i}\right)^{n-2} d v_{\imath}-\int_{\underline{v}}^{\bar{v}} v_{\imath} f\left(v_{\imath}\right) F\left(v_{i}\right)^{n-1} d v_{\imath} \\
\left.\quad=\frac{\bar{v}-v_{i}^{*}}{n(n-1)}+\frac{1}{n} \int_{\underline{v}}^{v_{i}^{*}} F\left(v_{\imath}\right)\right)^{n} d v_{i}-\int_{v_{i}^{*}}^{\bar{v}}\left[\frac{n F\left(v_{\imath}\right)^{n-1}-(n-1) F\left(v_{l}\right)^{n}}{n(n-1)}\right] d v_{\imath}>0,
\end{aligned}
$$


where the equality arises after integrating each term by parts, and the inequality holds since $n F^{n-1}-(n-1) F^{n} \leqslant 1$ for all $v_{1} \in\left[v_{i}^{*}, \underline{v}\right]$, so the first term dominates the third.

Q.E.D.

Proposition 2: A one-owner partnership $\left\{r_{1}=1, r_{2}=0, \ldots, r_{n}=0\right\}$ cannot be dissolved efficiently.

PROOF: For the $n$-player partnership $\left\{r_{1}=1, r_{2}=0, \ldots, r_{n}=0\right\}$, integrating by parts in (D) yields

$$
-\int_{\underline{v}}^{\bar{v}} F^{n-1} d v+\int_{\underline{v}}^{\bar{v}} F^{n} d v \geqslant 0,
$$

which fails for all finite $n$.

Q.E.D.

Proposition 3: Any partnership not owned by a single player can be dissolved efficiently for some distributions $F$.

Proof: The proof is by construction, using the distribution $F(u)=\left\{1-[u(v)]^{-\alpha}\right\} /\left\{1-T^{-\alpha}\right\}$ where $\alpha \in\left(0, \frac{1}{2}\right)$ and $u(v)=1+\{(T-1)(v-\underline{v}) /(\bar{v}-\underline{v})\}$ (so $u(\underline{v})=1$ and $\left.u(\bar{v})=T\right)$. Take an arbitrary partnership $\left\{r \mid r_{t}<1 \forall i\right\}$ and let $d=1-T^{-\alpha}$. Using a binomial expansion for $F^{n-2}$ and $F^{n-1}$ and performing the integration indicated in (D) yields

$$
\begin{aligned}
\phi(r)= & \left\{\left.\sum_{i=1}^{n} \frac{\alpha}{d^{n-1}}\left[\frac{u^{1-\alpha}}{1-\alpha}-(n-2) \frac{u^{1-2 \alpha}}{1-2 \alpha}+\cdots\right]\right|_{u=u\left(v_{i}^{*}\left(r_{t}\right)\right)} ^{T}\right. \\
& -\left.n \frac{\alpha}{d^{n}}\left[\frac{u^{1-\alpha}}{1-\alpha}-(n-1) \frac{u^{1-2 \alpha}}{1-2 \alpha}+\cdots\right]\right|_{u=1} ^{T}\left(\frac{\bar{v}-\underline{v}}{T-1}\right) .
\end{aligned}
$$

It suffices to show that the above terms in $T$ in the braces tend to $+\infty$ with $T$, because the terms in $T$ ignored are of lower order, and so are insignificant, and all the terms in the lower limits are finite because $u\left(v_{i}^{*}\left(r_{i}\right)\right)$ approaches a finite limit, $\forall r_{i}<1$. (It is crucial that we have $r_{i}<1$, since $u\left(v_{i}^{*}(1)\right)=T$, which does not stay finite as $T \rightarrow \infty$.) To show that the terms in $T$ approach $\infty$, replace $T$ by $(1-d)^{-1 / \alpha}$ and collect terms. This yields

$$
\frac{n \alpha}{d^{n-1}}\left((1-d)^{2-1 / \alpha}\left[\frac{1}{1-2 \alpha}-\frac{1}{d(1-\alpha)}\right]+\frac{(n-1)(1-d)^{3-1 / \alpha}}{d(1-2 \alpha)}\right) .
$$

As $T \rightarrow \infty, d \rightarrow 1$. Therefore, the second term above can be ignored, since it has an extra factor of $(1-d)$. Since $\alpha \in\left(0, \frac{1}{2}\right), 2-1 / \alpha<0$, so the first term goes to $\infty$ as $d \rightarrow 1$ provided

$$
\frac{1}{1-2 \alpha}-\frac{1}{d(1-\alpha)}>0
$$

which holds for $d \in((1-2 \alpha) /(1-\alpha), 1)$. So $\phi(r)>0$ for sufficiently large $T$.

Q.E.D.

Proposition 4: Given $F$ and an n-player partnership $\left\{r_{1}, \ldots, r_{n}\right\}$, there exists a finite $M$ such that for all $m>M$ the $m$-fold replication of the n-player partnership can be dissolved efficiently.

ProOF: By the concavity of $\phi$ established in Proposition 1, it suffices to show that the result holds for the $n$-player partnership $\left\{r_{1}=1, r_{2}=0, \ldots, r_{n}=0\right\}$, the $m$-fold replication of which is $\left\{r_{i}=1 / m, i=1, \ldots, m ; r_{j}=0, j=m+1, \ldots, m n\right\}$. Let $m n=N$. Then $m=N / n$ and the partnership of interest is $\left\{r_{1}=n / N, i=1, \ldots, N / n ; r_{j}=0, j=(N / n)+1, \ldots, N\right\}$. After integrating by parts in (D) and collecting terms, we have

$$
\begin{aligned}
\frac{1}{N} \phi(R)= & {\left[\frac{\bar{v}-v_{i}^{*}}{N(N-1)}-\int_{v_{i}^{*}}^{\bar{v}}\left(\frac{N F^{N-1}-(N-1) F^{N}}{N(N-1)}\right) d u\right] } \\
& +\left[\frac{1}{n} \int_{\underline{v}}^{v_{i}^{*}} \frac{F^{N-1}}{N-1} d u-\int_{\underline{v}}^{v^{*} \cdot}\left(\frac{N F^{N-1}-(N-1) F^{N}}{N(N-1)}\right) d u\right],
\end{aligned}
$$

where $v_{i}^{*}=v_{i}^{*}(n / N)=F^{-1}\left[(n / N)^{1 / N-1}\right]$. Since $N F^{N-1}-(N-1) F^{N} \leqslant 1$ over $\left[v_{i}^{*}, \bar{v}\right]$, the first pair of terms is strictly positive. The second pair is positive for sufficiently large $N$ if

$$
\lim _{N \rightarrow \infty} \frac{(N-1) \int_{v}^{v_{i}^{*}} F^{N} d u}{N \int_{\underline{v}}^{v_{i}^{*}} F^{N-1} d u}>1-\frac{1}{n} .
$$


Consider two arbitrary values $k_{1}<k_{2}$ from $(\underline{v}, \bar{v})$, and let $K_{1}=F\left(k_{i}\right)$ for $i=1,2$. As $N \rightarrow \infty, v_{i}^{*} \rightarrow \bar{v}$, so for sufficiently large $N$ we have $k_{1}<k_{2}<v_{1}^{*}$. Let $I=\int_{k_{1}^{2}}^{k_{2}} F^{N} d u$ and $J=\int_{k_{2}^{*}}^{v_{1}^{*}} F^{N} d u$. Then $\int_{k_{1}^{2}}^{k_{2}} F^{N-1} d u<I / K_{1}$ and $\int_{k_{2}^{*}}^{v_{1}^{*}} F^{N-1} d u<J / K_{2}<J / K_{1}$. So

$$
\begin{aligned}
\frac{\int_{v}^{v^{*}} F^{N} d u}{\int_{v}^{v^{*}} F^{N-1} d u} & >\frac{\int_{k_{1}^{2}}^{k_{2}} F^{N} d u+\int_{k_{2}^{\prime}}^{v^{*}} F^{N} d u}{\left(k_{1}-\underline{v}\right) K_{1}^{N-1}+\int_{k_{1}^{2}}^{k_{2}} F^{N-1} d u+\int_{k_{2}^{\prime}}^{v^{*}} F^{N-1} d u} \\
& >K_{1} \frac{I+J}{\left(k_{1}-\underline{v}\right) K_{1}^{N}+I+J} .
\end{aligned}
$$

Since $I+J>J>\left(v_{1}^{*}-k_{2}\right) K_{2}^{N}$, we have

$$
\begin{aligned}
K_{1} \frac{I+J}{\left(k_{1}-\underline{v}\right) K_{1}^{N}+I+J} & >K_{1} \frac{\left(v_{i}^{*}-k_{2}\right) K_{2}^{N}}{\left(k_{1}-\underline{v}\right) K_{1}^{N}+\left(v_{1}^{*}-k_{2}\right) K_{2}^{N}} \\
& =K_{1} \frac{v_{i}^{*}-k_{2}}{\left(k_{1}-\underline{v}\right)\left(K_{1} / K_{2}\right)^{N}+\left(v_{1}^{*}-k_{2}\right)} .
\end{aligned}
$$

Fix $\varepsilon>0$, and let $K_{1}=1-\frac{1}{2} \varepsilon$, and $K_{2}=1-\frac{1}{4} \varepsilon$. Then $\left(K_{1} / K_{2}\right)^{N} \rightarrow 0$ while $v_{1}^{*}-k_{2} \rightarrow \bar{v}-k_{2}$, so the last ratio above approaches $1-\frac{1}{2} \varepsilon$, and can therefore be made to exceed $1-(1 / n)$ for fixed $n$, as required.

Q.E.D.

PROPOSITION 5: A $k+1$-price auction has a symmetric equilibrium bidding strategy given by

$$
b\left(v_{i}\right)=v_{1}-\frac{\int_{z}^{v_{i}} F^{-1}(k)[F(z)-k]^{n} d z}{\left[F\left(v_{i}\right)-k\right]^{n}}
$$

Proof: Let $G(x)=F(x)^{n-1}$. If $i$ conjectures that the $n-1$ others will use the strategy $b\left(v_{j}\right)$, then $i$ 's expected utility from bidding $b_{i}$ with valuation $v_{t}$ is

$$
\begin{aligned}
U_{l}\left(v_{l}, b_{i}\right)= & \int_{\underline{v}}^{b^{-1}\left(b_{1}\right)}\left(v_{t}-\frac{n-1}{n}\left[k b(x)+(1-k) b_{i}\right]\right) d G(x) \\
& +\int_{x=b^{-1}\left(b_{i}\right)}^{\bar{v}} \int_{y=\underline{v}}^{b^{-1}\left(b_{1}\right)} \frac{1}{n}\left[k b_{i}+(1-k) b(x)\right] d H(y \mid x) d G(x) \\
& +\int_{x=b^{-1}\left(b_{t}\right)}^{\bar{v}} \int_{y=b^{-1}\left(b_{1}\right)}^{x} \frac{1}{n}[k b(y)+(1-k) b(x)] d H(y \mid x) d G(x),
\end{aligned}
$$

where $H(y \mid x)=[F(y) / F(x)]^{n-2}$ (for $y \leqslant x$ ) is the distribution of the second-largest of the $n-1$ other bids given that the largest is $x$. The best response for $i$ therefore solves

$$
\frac{\partial U_{i}}{\partial b_{i}}=\left(v_{i}-b_{1}\right) g\left[b^{-1}\left(b_{i}\right)\right] \frac{d b^{-1}}{d b_{i}}-\frac{n-1}{n} F\left[b^{-1}\left(b_{i}\right)\right]^{n-2}\left(F\left[b^{-1}\left(b_{i}\right)\right]-k\right)=0 .
$$

The symmetric equilibrium $b\left(v_{t}\right)$ satisfies

$$
v_{t}-b\left(v_{t}\right)=\frac{1}{n} b^{\prime}\left(v_{i}\right) \frac{F\left(v_{1}\right)-k}{f\left(v_{1}\right)} .
$$

This linear differential equation can be solved using the integrating factor $\left[F\left(v_{i}\right)-k\right]^{n}$; the solution is a one-parameter family satisfying

$$
\left[v_{\imath}-b\left(v_{i}\right)\right]\left[F\left(v_{\iota}\right)-k\right]^{n}=\int_{c}^{v_{t}}[F(u)-k]^{n} d u .
$$

We choose $c$ to make the right-hand side equal to zero at $v_{t}=F^{-1}(k)$; otherwise, bids tend to $\pm \infty$ as $v_{t}$ approaches $F^{-1}(k)$ from above or below. This choice of $c$ yields the symmetric equilibrium

$$
b\left(v_{l}\right)=v_{t}-\frac{\int_{z=F^{-1}(k)}^{v_{i}}[F(z)-k]^{n} d z}{\left[F\left(v_{i}\right)-k\right]^{n}},
$$

and truth-telling occurs at $v_{l}=F^{-1}(k)$. Finally, since $b^{\prime}>0$, this equilibrium is ex-post efficient: the partner with the highest valuation receives the good with probability one. 
It remains, then, to verify interim individual rationality. That is, we want $\tilde{w}\left(v_{t}, r_{t}\right)=$ $U\left[v_{l}, b\left(v_{l}\right)\right]-r_{i} v_{i} \geqslant 0$ for all $v_{t} \in[\underline{v}, \bar{v}]$, where $U\left[v_{l}, b\left(v_{l}\right)\right]$ is

$$
\begin{aligned}
& \int_{\underline{v}}^{v_{1}}\left(v_{1}-\frac{n-1}{n}\left[k b(x)+(1-k) b\left(v_{i}\right)\right]\right) d G(x) \\
& \quad+\int_{x=v_{t}}^{\bar{v}} \int_{y=\underline{v}}^{v_{1}} \frac{1}{n}\left[k b\left(v_{i}\right)+(1-k) b(x)\right] d H(y \mid x) d G(x) \\
& \quad+\int_{x=v_{t}}^{\bar{v}} \int_{y=v_{t}}^{x} \frac{1}{n}[k b(y)+(1-k) b(x)] d H(y \mid x) d G(x) .
\end{aligned}
$$

Partially differentiating with respect to $v_{i}$ and applying the first-order condition shows that, for fixed $r_{i}, \tilde{w}$ is minimized at $v_{i}^{*}\left(r_{t}\right)=G^{-1}\left(r_{i}\right)$. Let $w\left(r_{t}\right)=\tilde{w}\left[v_{i}^{*}\left(r_{t}\right), r_{i}\right]$; then

$$
\begin{aligned}
\frac{n}{n-1} w\left(r_{i}\right)= & (1-k)\left\{\frac{1}{n-1} \int_{v_{i}^{*}}^{\bar{v}} b(x) d G(x)-r_{i} b\left(v_{i}^{*}\right)\right\} \\
& +k\left\{F\left(v_{i}^{*}\right)^{n-2}\left[1-F\left(v_{\imath}^{*}\right)\right] b\left(v_{i}^{*}\right)-\int_{\underline{v}}^{v_{i}^{*}} b(x) d G(x)\right. \\
& \left.+\frac{1}{n-1} \int_{x=v_{i}^{*}}^{\bar{v}} \int_{y=v_{i}^{*}}^{x} b(y) d H(y \mid x) d G(x)\right\} .
\end{aligned}
$$

PROPOSITION 6: The set of partnerships that can be dissolved efficiently using a $k+1$-price auction is a nonempty, convex, symmetric subset of the simplex and is centered around the equal-shares partnership $(1 / n, \ldots, 1 / n)$.

Proof: Convexity follows from the concavity of $w\left(r_{t}\right)$, which holds because $w^{\prime}\left(r_{1}\right)=-v_{1}^{*}\left(r_{t}\right)$ and $w^{\prime \prime}\left(r_{i}\right)=-d / d r_{i} v_{i}^{*}\left(r_{i}\right)<0$ since $v_{i}^{*}=F^{-1}\left(r_{i}^{1 /(n-1)}\right)$. Symmetry follows from relabeling the partners. Finally consider the equal-shares partnership in two steps. First, consider the terms involving $1-k$. We have

$$
\frac{1}{n-1} \int_{v_{i}^{*}}^{\bar{v}} b(x) d G(x) \geqslant r_{i} b\left(v_{i}^{*}\right)
$$

at $v_{t}^{*}=G^{-1}(1 / n)$ by substituting $b\left(v_{i}^{*}\right)$ for $b(x)$ in the integral and simplifying. And second, consider the terms involving $k$. We have

$$
F\left(v_{i}^{*}\right)^{n-2}\left[1-F\left(v_{i}^{*}\right)\right] b\left(v_{i}^{*}\right) \geqslant \int_{\underline{v}}^{v_{i}^{*}} b(x) d G(x)-\frac{1}{n-1} \int_{x=v_{i}^{*}}^{\bar{v}} \int_{y=v_{i}^{*}}^{x} b(y) d H(y \mid x) d G(x)
$$

at $v_{i}^{*}=G^{-1}(1 / n)$, again by substituting $b\left(v_{i}^{*}\right)$ for $b(x)$ and $b(y)$ in the integrals and simplifying.

Q.E.D.

Proposition 7: As $n \rightarrow \infty$, the only partnership that can be dissolved efficiently by a $k+1$-price auction is the equal-shares partnership $(1 / n, \ldots, 1 / n)$. That is, letting $\rho_{n}$ be the largest share in a n-player partnership that any partner can have such that the partnership can be dissolved efficiently, $\rho_{n} /(1 / n) \rightarrow 1$ as $n \rightarrow \infty$.

Proof: It suffices to show that given $\delta>0$, there exists $N$ such that for all $n>N$, interim individual rationality fails for a player with share $(1+\delta) / n$. Let

$$
d(\delta)=F\left[\bar{v}\left(\frac{1+\frac{1}{2} \delta}{1+\delta}\right)\right]<1 .
$$


Interim individual rationality for a player with share $(1+\delta) / n$ and valuation $\bar{v}\left(\left(1+\frac{1}{2} \delta\right) /(1+\delta)\right.$ implies

$$
\begin{aligned}
{\left[\bar{v}\left(\frac{1+\frac{1}{2} \delta}{1+\delta}\right)\right]\left[\left(\frac{1+\delta}{n}\right)\right] \leqslant } & (\text { probability of losing })(\text { value of losing }) \\
& +(\text { probability of winning })(\text { value of winning) } \\
& <(1)\left(\frac{\bar{v}}{n}\right)+\left(d^{n-1}\right)(\bar{v}) .
\end{aligned}
$$

Thus,

$$
\left(1+\frac{1}{2} \delta\right)<1+n d^{n-1}
$$

which is necessarily false for all sufficiently large $n$.

Q.E.D.

\section{REFERENCES}

Gresik, Thomas A., And Mark A. Satterthwaite (1985): “The Rate at Which a Simple Market Becomes Efficient as the Number of Traders Increases: An Asymptotic Result for Optimal Trading Mechanisms," Working Paper No. 641, Graduate School of Management, Northwestern University.

Holmstrom, Bengt, AND Roger B. MYerson (1983): "Efficient and Durable Decision Rules with Incomplete Information," Econometrica, 51, 1799-1819.

MYerson, Roger B. (1979): "Incentive Compatibility and the Bargaining Problem," Econometrica, 47, 61-73.

Myerson, Roger B., And Mark A. Satterthwaite (1983): "Efficient Mechanisms for Bilateral Trading," Journal of Economic Theory, 28, 265-281.

Samuelson, William (1985): "A Comment on the Coase Theorem," in Game Theoretic Models of Bargaining, edited by Alvin Roth. Cambridge: Cambridge University Press.

VAN DAMME, ERIC (1985): "Fair Allocation of an Indivisible Commodity," Working Paper, University of Technology, Delft, the Netherlands.

Wilson, Robert (1985): "Incentive Efficiency of Double Auctions," Econometrica, 53, 1101-1116. 


\section{ERRATUM}

IN THE ARTICLE by P. Cramton, R. Gibbons, and P. Klemperer, "Dissolving a Partnership Efficiently," Econometrica, 55 (1987), 615-632, there is an error in the statement of Lemma 3 and Lemma 4 (page 618). The difficulty is that the proof of Lemma 3 assumes that the worst-off type $v_{i}^{*}$ satisfies $S_{i}\left(V_{i}^{*}\right)=r_{i}$. The following additional assumption is sufficient to guarantee that $S_{i}\left(v_{i}^{*}\right)=r_{i}$ : assume that $S_{i}$ has range $[0,1]$. The error is isolated to these two lemmas, since the remainder of the paper considers ex post efficient sharing rules, which satisfy this full-range assumption. The authors are grateful to Preston McAfee for drawing attention to the error. 